\title{
Research Article \\ Effects of Botulinum Toxin Type A on Microvessels in Hypertrophic Scar Models on Rabbit Ears
}

\author{
Na Zhou $\left(\mathbb{D},{ }^{1}\right.$ Dongping Li $\mathbb{D}^{2},{ }^{2}$ Yanzhu Luo $\mathbb{D}^{1},{ }^{1}$ Junping $L i \mathbb{D}^{1},{ }^{1}$ and Yuhong Wang $\mathbb{D}^{2}$ \\ ${ }^{1}$ Aier School of Ophthalmology, Central South University, Changsha, China \\ ${ }^{2}$ Department of Oculoplastic Surgery, Hankou Aier Eye Hospital, Wuhan, China
}

Correspondence should be addressed to Yuhong Wang; wangyuhong10@hotmail.com

Received 17 February 2020; Accepted 26 May 2020; Published 16 June 2020

Academic Editor: Nicholas Dunne

Copyright ( $92020 \mathrm{Na}$ Zhou et al. This is an open access article distributed under the Creative Commons Attribution License, which permits unrestricted use, distribution, and reproduction in any medium, provided the original work is properly cited.

\begin{abstract}
Background. Although Botulinum Toxin Type A (BTXA) has been applied to scar prevention and treatment, the mechanisms still require further exploration. Objective. To investigate the effects of BTXA on microvessels in the hypertrophic scar models on rabbit ears. Methods. Eight big-eared New Zealand rabbits (males or females) were selected to establish scar models. One ear of each rabbit (4 models in each ear) was selected randomly to be injected with BTXA immediately after modeling and included in the treated group, while the opposite ear was untreated and included in the control group. The growth of scars in each group was observed and recorded, and 4 rabbits were sacrificed on days 30 and 45 after modeling. Then, scar height was measured by hematoxylin-eosin (HE) staining, vascular endothelial growth factor (VEGF) expression was detected by immunohistochemical (IHC) testing, and microvessel density (MVD) was calculated based on CD34 (human hematopoietic progenitor cell antigen). Results. The wounds in each group were well healed and free from infection or necrosis. On days 30 and 45, the scar height, MVD value, and VEGF expression in the treated group were lower than those in the control group $(P<0.05)$. For the treated group, the above indicators on day 45 were lower than on day $30(P>0.05)$. Besides, there was a positive correlation between the MVD value and the VEGF expression in the treated group $(P<0.05)$. Conclusion. The injection of BTXA immediately after modeling inhibits VEGF expression and reduces angiogenesis, thereby inhibiting hypertrophic scar formation.
\end{abstract}

\section{Introduction}

As a pathological scar generally caused by burns, injuries, and surgery, the hypertrophic scar (HS) can affect the life quality of patients both physically and psychologically, for which reason it has long been a great challenge for clinicians. In recent years, BTXA has been successively reported to be effective in scar prevention and treatment [1-6], but the studies on related mechanisms mainly focus on BTXA inhibiting fibroblast generation, promoting apoptosis, and reducing collagen deposition $[7,8]$, and there were few reports on its effects on microvessels. However, as studies have shown that the hyperplasia of scar tissue is closely related to MVD and microcirculatory blood flow [9], could BTXA be effective in scar prevention and treatment by inhibiting angiogenesis? This study was performed to determine the effects of BTXA on blood vessels in HS by detecting the VEGF expression and MVD in the HS on rabbit ears injected with BTXA.

\section{Materials and Methods}

2.1. Subjects. Eight big-eared New Zealand rabbits (males or females) in healthy condition with sound ears, weighing $2-3 \mathrm{~kg}$, were selected as subjects from the Laboratory Animal Center of Tongji Medical College, Huazhong University of Science and Technology. They were fed regularly in separate cages at an indoor temperature of $21-25^{\circ} \mathrm{C}$ with clean air ventilation in accordance with the Policy on Humane Care and Use of Laboratory Animals.

2.2. Reagents. The reagents are as follows: hematoxylin (Sigma), eosin Y (water-soluble), absolute ethanol, xylene, 
neutral balsam (Sinopharm Chemical Reagent Co., Ltd.), PBS solution $(0.01 \mathrm{M})$, hematoxylin stain solution, $\mathrm{H}_{2} \mathrm{O}_{2}$, EDTA ( $\mathrm{pH}$ 8.0) antigen retrieval solution (Wuhan Hundred Thousand Degree Biological Technology Co., Ltd.), BSA (bovine serum albumin) (BIOFROXX), DAB substrate kit (DAKO), VEGF primary antibodies (Proteintech Group), CD34 primary antibodies (BioWorld), and IHC secondary antibody kit (SeraCare).

\subsection{Experimental Protocol}

2.3.1. Modeling. The rabbits were raised for one week before the study for acclimatization, fasted and forbidden to drink for $8 \mathrm{~h}$ before modeling, and then anesthetized with $3 \%$ pentobarbital sodium $(30 \mathrm{mg} / \mathrm{kg})$. According to the method proposed by Morris and $\mathrm{Wu}[10]$, the ventral side of a rabbit ear was disinfected with complex iodine 3 times; the fullthickness skin and perichondrium were drilled through with a $6 \mathrm{~mm}$ diameter ophthalmic corneal trephine; with the skin and perichondrium fully removed and the cartilage preserved, the wound was first pressed to stop the bleeding, then exposed after cleaning with normal saline. A total of 4 identical wounds were prepared on both sides along the long axis on each rabbit ear at an interval of $1.5-2 \mathrm{~cm}$.

2.3.2. Grouping. One ear of each rabbit was randomly selected to be injected with BTXA and included in the treated group, while the opposite ear was untreated and included in the control group. The growth of scars in each group was observed and recorded every 2 days, and 4 rabbits were sacrificed on days 30 and 45 after modeling.

\subsubsection{Method}

(1) Injection and Sampling. BTXA (Lanzhou Biochemical Company, Lanzhou City, China), diluted with normal saline to $40 \mathrm{IU} \cdot \mathrm{mL}^{-1}$, was subcutaneously injected with $30 \mathrm{G}$ needles at 3 and 9 o'clock on each wound approx. $3 \mathrm{~mm}$ under the skin at a dose of $2 \mathrm{U} /$ point. The general condition of the wounds was observed every 1-2 days, and sampling covered scar tissue and surrounding normal tissues within a range of at least $2 \mathrm{~mm}$.

(2) Histological Examination. Tissue specimens were fixed in $4 \%$ paraformaldehyde, dehydrated in ethanol at different gradients until transparent, and then embedded in paraffin, sliced into serial sections at $5 \mu \mathrm{m}$ thickness, and finally processed for HE staining and IHC staining.

Scar height: this refers to the highest vertical height between the epidermis at the top of the bulging scar tissue and cartilage, which was measured by a light microscope after HE staining.

MVD calculation: in the 3 views randomly selected from each section, the presence of brownish-yellow granules in the cytoplasms of VECs was considered as CD34-positive. Referring to the sampling and counting method of Weidner [11], the number of positive-stained vessels was counted under a $200 \times$ light microscope, and the mean value of the 3 views was counted as the MVD value (/scope).
VEGF expression detection: photos with identical brownish-yellow color were selected by Image-Pro Plus 6.0 (Media Cybernetics, Inc., Rockville, MD, USA) as the uniform criterion for judging the positivity of all photos, thus analyzing the integrated optical density (IOD) of the positivity of each photo.

2.4. Statistical Analysis. The measurement data was expressed as mean \pm standard deviation $(X \pm s)$ and processed for statistical analysis using SPSS 18.0 statistical software. The Shapiro-Wilk test was applied to determine normality, and Levene's test was applied to assess homogeneity of variance. The scar height, MVD value, and VEGF expression were compared between the treated group and the control group using the $t$-test of two independent samples, the correlation between the two variables was determined using Pearson linear correlation analysis, and $P<0.05$ indicated statistically significant difference.

\section{Results}

3.1. Gross Observation. The wounds in each group were completely healed in about 15-19 days and free from infection or necrosis. On days 30 and 45, the scars in the treated group were flatter than those in the control group and soft to the touch (Figure 1).

3.2. Scar Height. On days 30 and 45, the scar height of the treated group was lower than that of the control group, and the difference was statistically significant $(P<0.05)$; the scar height of the treated group on day 45 was lower than that on day $30(t=1.220, P=0.232)$ (Table 1 , Figure 2$)$.

3.3. MVD. On days 30 and 45, the MVD value of the treated group was less than that of the control group, and the difference was statistically significant $(P<0.05)$; the MVD value of the treated group on day 45 was less than that on day 30 $(t=1.602, P=0.127$ ) (Table 2, Figures 3 and $4(\mathrm{a})$ and $4(\mathrm{~b}))$.

3.4. VEGF. On days 30 and 45, the VEGF expression of the treated group was less than that of the control group, and the difference was statistically significant $(P<0.05)$; the VEGF expression of the treated group on day 45 was less than that on day $30(t=1.379, P=0.185)$ (Table 3 , Figures $4(\mathrm{c})$ and $4(\mathrm{~d})$ and 5).

3.5. Correlation Analysis. There was a positive correlation between MVD value and VEGF expression in the treated group on day $30(r=0.671, P<0.05)$ and day $45(r=0.732$, $P<0.05)$ (Figure 6).

\section{Discussion}

Although scarring is inevitable in regenerative repair and wound healing after tissue injury, HS incidence is extremely high, ranging from $40 \%$ to $91 \%$ for various reasons [12], and Asian people tend to form scars after skin injury [13]. It is widely believed that HS, a pathological scar, is formed due to blocked apoptosis and excessive generation of fibroblast, decreased degradation and increased synthesis of 


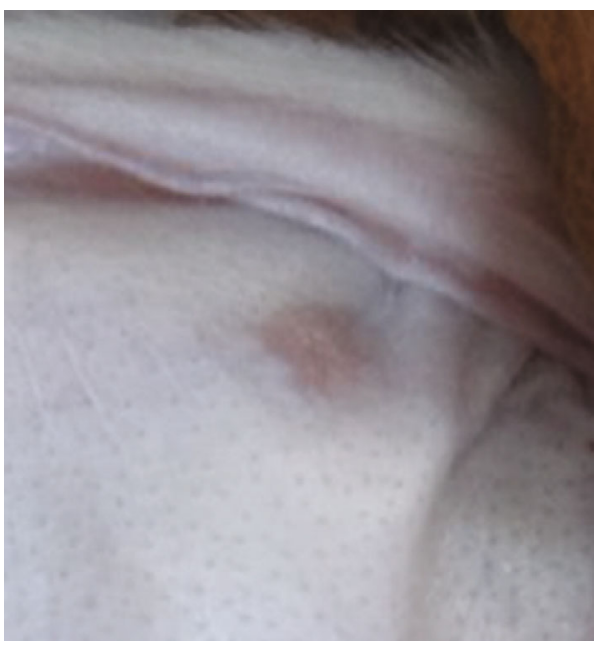

(a)

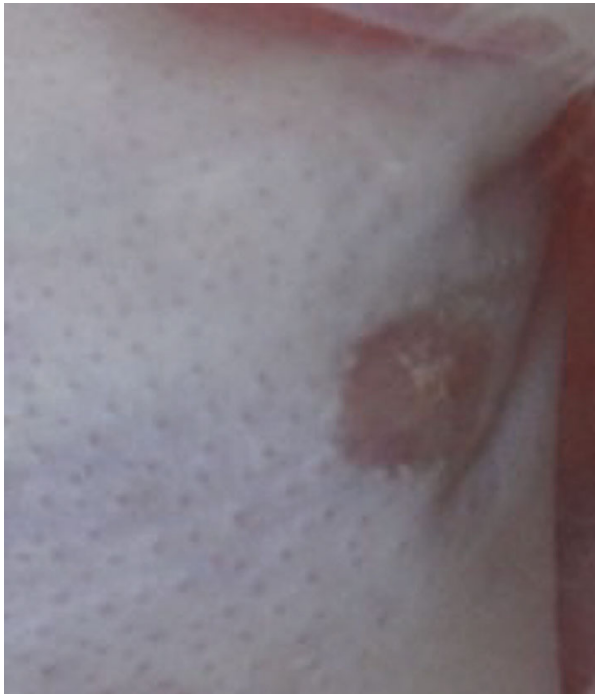

(b)

Figure 1: Gross appearance. (a) Gross appearance of treated group at day 45. (b) Gross appearance of control group at day 45.

TABLE 1: Scar height of two groups at each time point (mm).

\begin{tabular}{lcc}
\hline & Day 30 & Day 45 \\
\hline Treated & $0.59 \pm 0.07$ & $0.56 \pm 0.07$ \\
Control & $1.12 \pm 0.08$ & $0.98 \pm 0.07$ \\
$t$ & -19.153 & -16.171 \\
$p$ & 0.000 & 0.000 \\
\hline
\end{tabular}

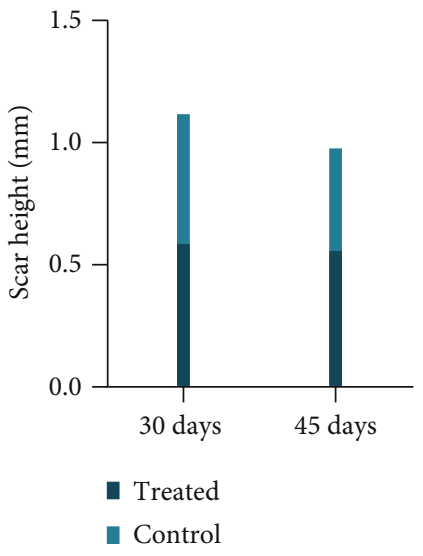

Figure 2: Scar height of two groups at each time point.

TABLE 2: MVD value of two groups at each time point (/scope).

\begin{tabular}{lcc}
\hline & Day 30 & Day 45 \\
\hline Treated & $57.43 \pm 15.27$ & $48.33 \pm 9.46$ \\
Control & $94.20 \pm 19.45$ & $78.50 \pm 12.01$ \\
$t$ & -4.703 & -6.239 \\
$p$ & 0.000 & 0.000 \\
\hline
\end{tabular}

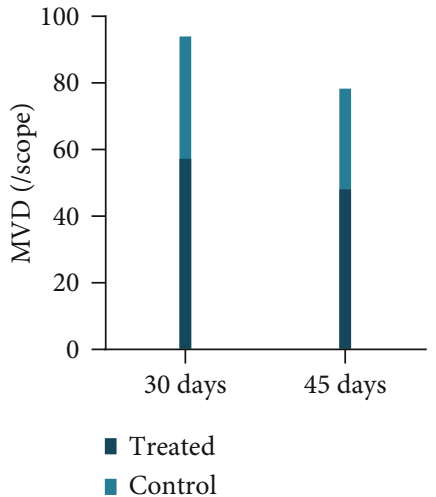

Figure 3: MVD value of two groups at each time point.

extracellular matrix collagen, and disturbed regulation of various cytokines [14].

In previous studies of BTXA injection in different periods, it was found that the effect of BTXA injection immediately after modeling was equivalent to that of the injection 10 days after modeling, with wound healing unaffected. Specifically, the latter was more effective in reducing the number of fibroblasts, while the former could significantly reduce the number of microvessels in the scar tissue according to HE staining. Thus, the study was aimed at further verifying the effects of BTXA on microvessels in HS tissues (unpublished data).

As angiogenesis is essential to wound healing, and multiple stages benefit from the participation of blood vessels and their bioactivators, angiogenesis and revascularization are critical for wound healing [15]. Angiogenesis can be quantified by MVD instead of direct measurement. CD34, a $110 \mathrm{kDa}$ transmembrane glycoprotein of unknown function, is considered an important marker of angiogenesis and can represent MVD in tissues [16]. VEGF is the most potent angiogenic cell growth factor which is known to stimulate 


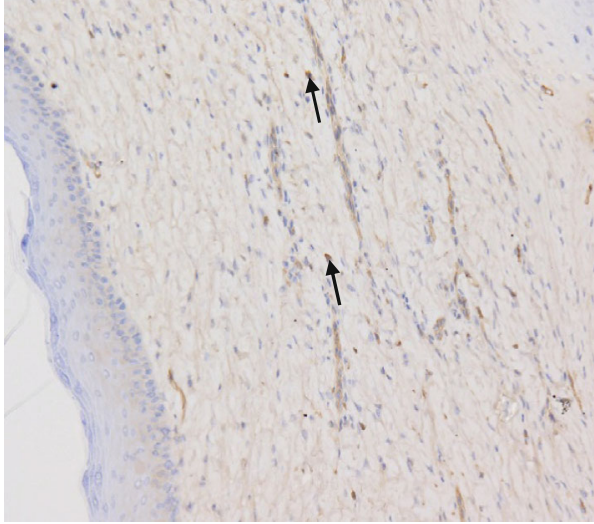

(a)

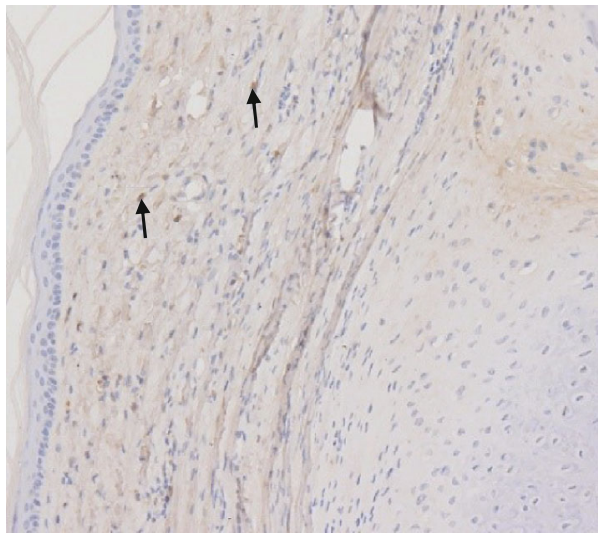

(c)

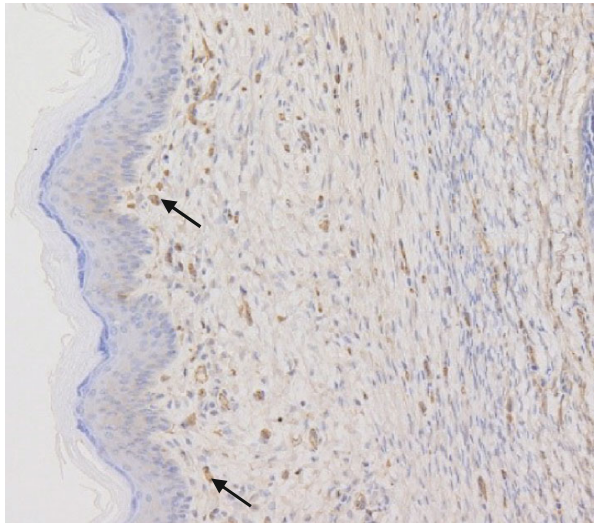

(b)

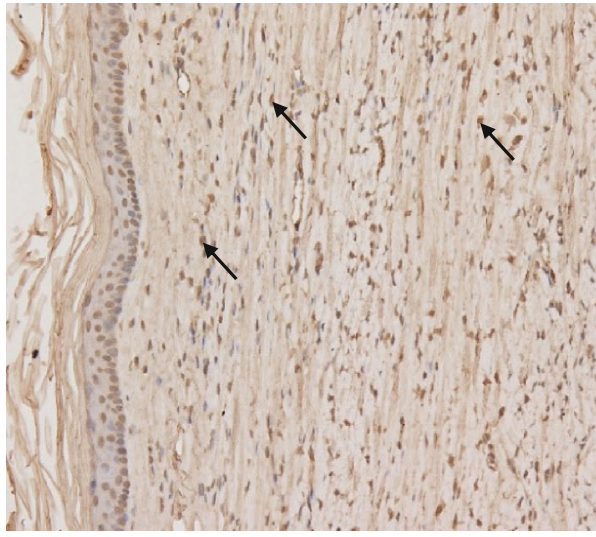

(d)

FIgURE 4: Expression of CD34 and VEGF $(\times 200)$. $(\mathrm{a}, \mathrm{b})$ are positive expressions of CD34 in the treated group and the control group, respectively (as shown by the black arrow). (c, d) are positive expressions of VEGF in the treated group and the control group, respectively (as shown by the black arrow).

TABLE 3: VEGF-positive IOD value of two groups at each time point.

\begin{tabular}{lcc}
\hline & Day 30 & Day 45 \\
\hline Treated & $916.20 \pm 199.35$ & $797.93 \pm 183.99$ \\
Control & $2724.39 \pm 988.03$ & $2480.58 \pm 940.73$ \\
$t$ & -5.673 & -5.551 \\
$p$ & 0.000 & 0.000 \\
\hline
\end{tabular}

the migration of endothelial cells and increase vascular permeability, playing an extremely important role in angiogenesis and tissue repair $[17,18]$. The aberrant hyperplasia of local microvessels is an important factor in HS formation, and VEGF and CD105 are highly expressed in pathological scar tissue, suggesting that abnormal vascular hyperplasia is involved in the development of pathological scars [18-20]. In addition, related studies have also confirmed that the degree of scar hyperplasia is related to microvessel flow [21].

According to the results of the study, the scar height, VEGF expression, and MVD value in scar tissue were all reduced in the BTXA-injected group, suggesting that the inhibition of scars by BTXA is related to the reduction of

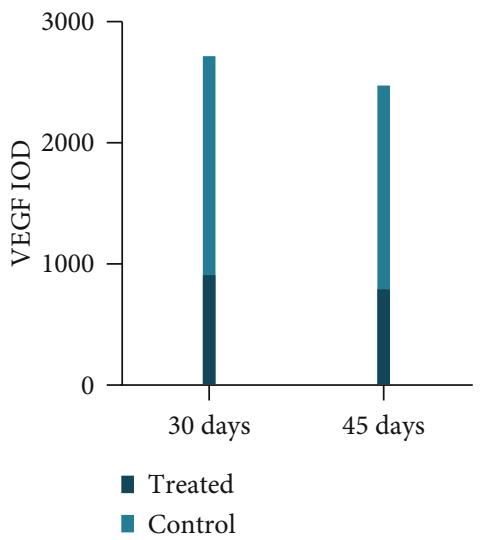

FIGURE 5: VEGF-positive IOD value of two groups at each time point.

angiogenesis. By using models of scarless and fibrotic repair, Wilgus et al. [22] confirmed that fibrotic wounds had relatively more blood vessels with higher VEGF expression and that neutralizing VEGF could reduce vascularity and inhibit scar formation. VEGF may also regulate scar formation indirectly by stimulating endothelial cells or affecting inflammatory cells [23]. 


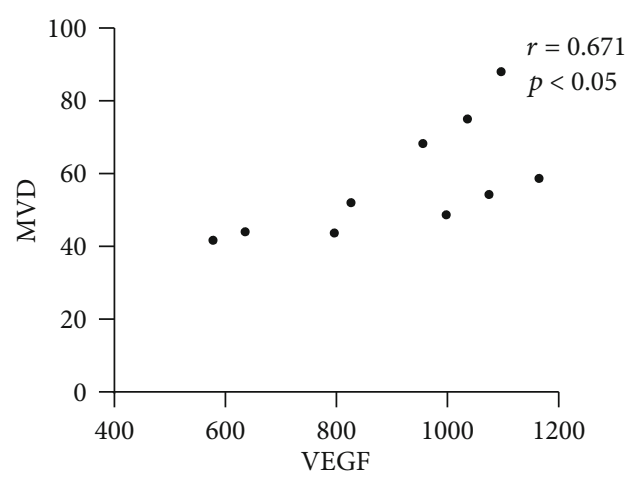

(a)

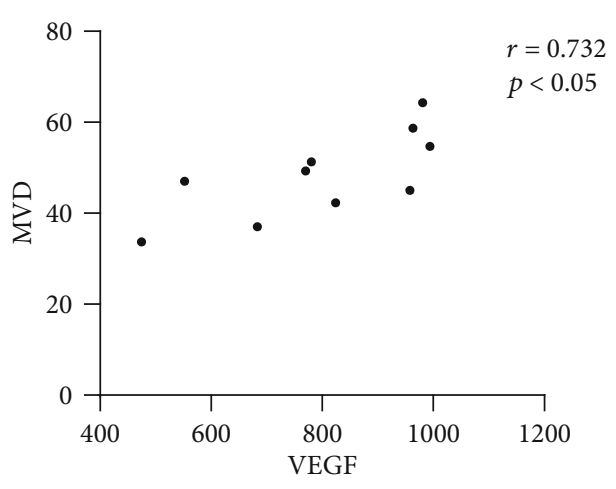

(b)

FIgURE 6: Correlation analysis of VEGF and MVD. (a) Correlation analysis of VEGF and MVD in the 30-day treated group. (b) Correlation analysis of VEGF and MVD in the 45-day treated group.

Arnold et al. [24] found that the VEGF-165 mRNA expression was decreased in the BTXA-injected group compared with the saline-injected group in a rat pedicled abdominal flap model study, while VEGF-165 mRNA was positively correlated with angiogenesis [25]. A significant correlation was found between CD34 and VEGF in an oral submucous fibrosis study by Sharma et al. [26], which was similar to the results herein. This study also revealed that the scar height, MVD value, and VEGF expression in the treated group on day 45 tended to be lower than those on day 30, which may be related to the decreased VEGF expression, vasoconstriction, occlusion, and chronic remodeling of scar tissue in the late stages of wound healing, while BTXA may be mainly effective in early healing.

At present, there are some studies on the effects of BTXA on VEGF and angiogenesis. Specifically, Eliane et al. [27] found that the distribution of proteoglycans and glycosaminoglycans, as well as VEGF expression, was reduced wherever BTXA was injected in the masseter muscles of transgenic mice. Similarly, Che et al. [28] established a rat model of supraspinatus tendon injury and injected BTXA into the supraspinatus muscle after modeling. The BTXAinjected group was compared with the saline group 4 weeks after injection, and VEGF expression in the BTXA-injected group was found to be significantly lower. Zhou et al. [29] established a rat model of prostatic hyperplasia and injected BTXA into the prostate tissue. BTXA was then observed to inhibit VEGF expression, thereby inhibiting angiogenesis in the prostate tissue and promoting prostate shrinkage, which was consistent with the results of this study.

However, the correlation of botulinum toxin versus VEGF and angiogenesis is still controversial. Some previous studies demonstrated conflicting results. Park and Park [30] demonstrated that BTXA treatment might increase the expression of VEGF and yield protection against ischemiareperfusion injury depending on increasing angiogenesis. Other studies also concluded that the group with BTXA treatment had higher VEGF expression in a rat transplantation model or transverse rectus abdominis myocutaneous flap in a rat model $[31,32]$. The concentration of BTXA used in these studies was $10 \mathrm{IU} / \mathrm{mL}$, but Gugerell et al. [33] found that high BTXA concentration of $20 \mathrm{IU} / \mathrm{mL}$ might reduce
VEGF expression and inhibit angiogenesis. In addition, Harper and Bates [34] highlighted that VEGF has a proangiogenic isoform and an antiangiogenic isoform, and the antiangiogenic VEGFxxxb isoforms might be switched to the proangiogenic VEGFxxx isoforms via splicing factors [35]. Thus, the VEGF isoform balance and the BTXA concentration might cause the conflicting result of correlation between BTXA and VEGF, which still need further research.

\section{Conclusion}

These results indicate that the injection of BTXA immediately after modeling can inhibit VEGF expression and reduce angiogenesis in tissues, thereby inhibiting HS formation in rabbit ears. However, more ample research is still needed to investigate the biology effects of BTXA on microvessels during the process of wound healing.

\section{Data Availability}

The data used to support the findings of this study are available from the corresponding author upon request.

\section{Conflicts of Interest}

The authors declared no potential conflicts of interest with respect to the research, authorship, and publication of this article.

\section{Acknowledgments}

This study was funded by the Aier Eye Hospital Group (grant number AF1510D13).

\section{References}

[1] C.-S. Chang, C. G. Wallace, Y.-C. Hsiao, C.-J. Chang, and P. K.-T. Chen, "Botulinum toxin to improve results in cleft lip repair," Plastic and Reconstructive Surgery, vol. 134, no. 3, pp. 511-516, 2014.

[2] M. Ziade, S. Domergue, D. Batifol et al., "Use of botulinum toxin type A to improve treatment of facial wounds: a 
prospective randomised study," Journal of Plastic, Reconstructive \& Aesthetic Surgery, vol. 66, no. 2, pp. 209-214, 2013.

[3] Y. S. Kim, H. J. Lee, S. H. Cho, J. D. Lee, and H. S. Kim, "Early postoperative treatment of thyroidectomy scars using botulinum toxin: a split-scar, double-blind randomized controlled trial," Wound Repair and Regeneration, vol. 22, no. 5, pp. 605-612, 2014.

[4] Y.-H. Li, J. Yang, J.-Q. Liu et al., “A randomized, placebo-controlled, double-blind, prospective clinical trial of Botulinum toxin type a in prevention of hypertrophic scar development in median sternotomy wound," Aesthetic Plastic Surgery, vol. 42, no. 5, pp. 1364-1369, 2018.

[5] H. G. Gassner, A. E. Brissett, C. C. Otley et al., "Botulinum toxin to improve facial wound healing: a prospective, blinded, placebo-controlled study," Mayo Clinic Proceedings, vol. 81, no. 8, pp. 1023-1028, 2006.

[6] L. Hu, Y. Zou, S. J. Chang et al., "Effects of botulinum toxin on improving facial surgical scars: a prospective, split-scar, double-blind, randomized controlled trial," Plastic and Reconstructive Surgery, vol. 141, no. 3, pp. 646-650, 2018.

[7] Z. Xiao and G. Qu, "Effects of botulinum toxin type A on collagen deposition in hypertrophic scars," Molecules (Basel, Switzerland), vol. 17, no. 2, pp. 2169-2177, 2012.

[8] X. zhibo and Z. miaobo, "Botulinum toxin type A affects cell cycle distribution of fibroblasts derived from hypertrophic scar," Journal of Plastic, Reconstructive \& Aesthetic Surgery, vol. 61, no. 9, pp. 1128-1129, 2008.

[9] Y. Zhang and D. Peng, "Progress of study on the blood capillary construction of hyperplasia of scar tissue," Asia-Pacific Traditional Medicine, vol. 3, no. 10, pp. 29-32, 2007.

[10] D. E. Morris, L. Wu, L. L. Zhao et al., "Acute and chronic animal models for excessive dermal scarring: quantitative studies," Plastic and Reconstructive Surgery, vol. 100, no. 3, pp. 674-681, 1997.

[11] N. Weidner, "Current pathologic methods for measuring intratumoral microvessel density within breast carcinoma and other solid tumors," Breast Cancer Research and Treatment, vol. 36, no. 2, pp. 169-180, 1995.

[12] G. G. Gauglitz, H. C. Korting, T. Pavicic, T. Ruzicka, and M. G. Jeschke, "Hypertrophic scarring and keloids: pathomechanisms and current and emerging treatment strategies," Molecular Medicine (Cambridge, Mass), vol. 17, no. 1-2, pp. 113-125, 2011.

[13] S. Kim, T. H. Choi, W. Liu, R. Ogawa, J. S. Suh, and T. A. Mustoe, "Update on scar management," Plastic and Reconstructive Surgery, vol. 132, no. 6, pp. 1580-1589, 2013.

[14] F. Xiaobin and B. Cheng, "Further importance to mechanism of pathological scar," Chinese Journal of Reparative and Reconstructive Surgery, vol. 1, pp. 1-5, 2005.

[15] W. M. van der Veer, F. B. Niessen, J. A. Ferreira et al., "Time course of the angiogenic response during normotrophic and hypertrophic scar formation in humans," Wound Repair and Regeneration, vol. 19, no. 3, pp. 292-301, 2011.

[16] R. S. Desai, G. S. Mamatha, M. J. Khatri, and S. J. Shetty, "Immunohistochemical expression of CD34 for characterization and quantification of mucosal vasculature and its probable role in malignant transformation of atrophic epithelium in oral submucous fibrosis," Oral Oncology, vol. 46, no. 7, pp. 553-558, 2010.

[17] D. O. Bates, "Vascular endothelial growth factors and vascular permeability," Cardiovascular Research, vol. 87, no. 2, pp. 262$271,2010$.
[18] Y. Li, X. Long, and Y. Li, "Changes and significance of VEGF and CD105 expression in pathological scar tissue," Shandong Medical Journal, vol. 22, pp. 67-68, 2015.

[19] R. Huang, J. Liang, and Z. Wu, "Correlation between platelet derived growth factor and angiogenesis in pathological scars," Guangdong Medical Journal, vol. 32, no. 4, pp. 457-460, 2011.

[20] L. Pan, L. Tu, and J. Luo, "Study on the inhibition of skin scar formation by selective cyclooxygenase- 2 inhibitor nimesulide," Journal of Clinical Rehabilitative Tissue Engineering Research, vol. 33, pp. 6137-6142, 2012.

[21] L. Bussche, R. M. Harman, B. A. Syracuse et al., "Microencapsulated equine mesenchymal stromal cells promote cutaneous wound healing in vitro," Stem Cell Research \& Therapy, vol. 6, no. 1, article 66, 2015.

[22] T. A. Wilgus, A. M. Ferreira, T. M. Oberyszyn, V. K. Bergdall, and L. A. DiPietro, "Regulation of scar formation by vascular endothelial growth factor," Laboratory Investigation, vol. 88, no. 6, pp. 579-590, 2008.

[23] T. A. Wilgus, "Vascular endothelial growth factor and cutaneous scarring," Advances in Wound Care, vol. 8, no. 12, pp. 671-678, 2019.

[24] P. B. Arnold, T. Fang, S. J. Songcharoen, G. Ziakas, and F. Zhang, "Inflammatory response and survival of pedicled abdominal flaps in a rat model after perivascular application of botulinum toxin type A," Plastic and Reconstructive Surgery, vol. 133, no. 4, pp. 491e-498e, 2014.

[25] L. Qing, Vascular endothelial growth factor 165 and neuropilin-l mRNA expression in esophageal squamous cell carcinoma and the association with angiogenesis, Zhengzhou University, 2005.

[26] E. Sharma, N. Tyagi, V. Gupta, A. Narwal, H. Vij, and D. Lakhnotra, "Role of angiogenesis in oral submucous fibrosis using vascular endothelial growth factor and CD34: an immunohistochemical study," Indian Journal of Dental Research, vol. 30, no. 5, pp. 755-762, 2019.

[27] E. H. Dutra, M. H. O’ Brien, A. Lima et al., "Cellular and matrix response of the mandibular condylar cartilage to botulinum toxin," PLoS One, vol. 11, no. 10, article e0164599, 2016.

[28] L. Che, Q. Zhou, T. Ye, C. Zhuang, P. Shang, and L. Wang, "Experimental study of stress loss effects on tendon-bone healing process after acute rotator cuff injury," Chinese Journal of Joint Surgery (Electronic Version), vol. 2, pp. 224-228, 2015.

[29] P. Zhou, X. Zhang, and Z. Xu, "Effect of botulinum toxin type A on expression of HIF- $1 \alpha$ and VEGF in rat prostatic tissue of hyperplasia," Zhejiang Medical Journal, vol. 5, pp. 401-404, 2014.

[30] T. H. Park and Y. J. Park, "The effect of botulinum toxin A on ischemia-reperfusion injury in a rat model," BioMed Research International, vol. 2017, Article ID 1074178, 7 pages, 2017.

[31] T. H. Park, S. H. Lee, Y. J. Park, Y. S. Lee, D. K. Rah, and S. Y. Kim, "Presurgical botulinum toxin a treatment increases angiogenesis by hypoxia-inducible factor- $1 \alpha /$ vascular endothelial growth factor and subsequent superiorly based transverse rectus abdominis myocutaneous flap survival in a rat model," Annals of Plastic Surgery, vol. 76, no. 6, pp. 723-728, 2016.

[32] Y. J. Park, J. W. Lee, Y. Chong, and T. H. Park, "Botulinum toxin A increases allograft tolerance in an experimental transplantation model: a preliminary study," Bioscience Reports, vol. 38, no. 2, 2018. 
[33] A. Gugerell, J. Kober, M. Schmid, E. Buchberger, L. P. Kamolz, and M. Keck, "Botulinum toxin A: dose-dependent effect on reepithelialization and angiogenesis," Plastic and Reconstructive Surgery - Global Open, vol. 4, no. 8, article e837, 2016.

[34] S. J. Harper and D. O. Bates, "VEGF-A splicing: the key to antiangiogenic therapeutics?" Nature Reviews Cancer, vol. 8, no. 11, pp. $880-887,2008$.

[35] Y. Qiu, C. Hoareau-Aveilla, S. Oltean, S. J. Harper, and D. O. Bates, "The anti-angiogenic isoforms of VEGF in health and disease," Biochemical Society Transactions, vol. 37, no. 6, pp. 1207-1213, 2009. 